\title{
Besoins perçus et adaptation des démarches d'enseignement: le cas de l'enseignement des sciences et technologies au secondaire
}

\author{
Sylvie Houde \\ Université de Sherbrooke \\ Jean-Claude Kalubi \\ Université de Sherbrooke
}

\begin{abstract}
Résumé
Cet article traite de l'adaptation des démarches d'enseignement en cours d'action, adoptées par les enseignants des sciences et technologies de l'école secondaire. Il vise à comprendre les processus dynamiques de l'adaptation de l'enseignement. Il s'appuie sur des données qui ont été obtenues grâce à un protocole de recherche qualitative axé sur des techniques de groupes de réflexion. Les résultats de la recherche mettent en évidence l'hétérogénéité des parcours d'adaptation des enseignants. Ils soulignent la nécessité du professionnalisme de l'enseignant des sciences et technologies. Ils suggèrent de prendre en considération la complexité des besoins d'adaptation. Ceux-ci commandent en définitive la compatibilité entre les démarches de l'enseignant et les besoins de ses élèves. Le processus d'adaptation est aussi guidé par le plaisir de la reconnaissance, les rapports de l'enseignant aux savoirs, de même que par la défense des valeurs collectives.
\end{abstract}

Mots clé: adaptation de l'enseignement, perception des besoins, enseignement des sciences et technologies

Sylvie Houde est conseillère pédagogique au Centre de pédagogie des sciences de la santé de la Faculté de médecine et des sciences de la santé de l'Université de Sherbrooke. Ses champs d'intérêt sont l'enseignement des sciences et technologies, l'adaptation des méthodes et stratégies d'enseignement dans différents contextes et la formation continue des professeurs.

Email: Sylvie.Houde@USherbrooke.ca

Jean-Claude Kalubi est professeur titulaire à la Faculté d'éducation de l'Université de Sherbrooke. Il assume les fonctions de directeur du Département d'études sur l'adaptation scolaire et sociale. Il est également titulaire de la Chaire de recherche sur les identités et innovations professionnelles. Ses travaux s'orientent vers développement des compétences et l'intervention auprès des élèves présentant des besoins spéciaux. 


\title{
Perceived needs and the adjustment of teaching approaches: the case of science and technology teaching in secondary schools
}

\author{
Sylvie Houde \\ Sherbrooke University \\ Jean-Claude Kalubi \\ Sherbrooke University
}

\begin{abstract}
This article deals with the adjustment of teaching approaches made by high school science and technology teachers while teaching. It aims at achieving a better understanding of the dynamic processes of teaching adjustment. It relies on a qualitative methodology using focus groups. Data analysis shows the multiplicity of ways that teachers use to adjust their teaching. It stresses out the importance of science and technology teachers' professionalism and suggests taking into account the complexity of teacher and student needs in the equation of teaching adjustment. Those needs call for an increased compatibility between teaching approaches and students' needs. The adjustment of teaching approaches is also related to the quest for recognition, the teacher relationship with knowledge and the collective values in place.
\end{abstract}

Keywords: Teaching adjustment, students and teachers needs, science and technology teaching

Sylvie Houde is pedagogical consultant and instructional designer at the Centre de pédagogie des sciences de la santé of the Faculty of Medicine and Health Sciences, Sherbrooke University. Her interests include science and technology teaching, adaptation of teaching methods and strategies in diverse settings.

Email: Sylvie.Houde@USherbrooke.ca

Jean-Claude Kalubi is professor at the Faculty of Education of Sherbrooke University, and director of the Department of Special Education. He also holds a research chair on professional identities and innovations. His work focuses on competency development and intervention with students with special needs.

Brock Education, Volume 19, No. 1, 


\section{Des besoins perçus et adaptation des démarches d'enseignement: le cas de l'enseignement des sciences et technologies au secondaire}

La formation en sciences et technologies (ST) se heurte à des critiques sévères, en raison des difficultés persistantes qu'elle rencontre. Pour Giordan (1999), le tableau des accusations n'a guère changé au cours des dernières décennies : « vingt ans plus tard, le constat est le même: l'enseignement des sciences reste inadapté. Quels savoirs transmettre et comment? La question garde toute son actualité et sa pertinence. Une prise de conscience s'impose » (p. 7). Pour nombre d'auteurs, l'école parviendrait difficilement à intéresser les élèves et à les inciter à poursuivre des études, voire une carrière dans les domaines liés aux ST (Conseil de la Science et de la Technologie [CST], 2002a; Reiss, 2004; Shapiro, 2004). À ce chapitre, le Québec ne fait pas exception. Même si les recherches démontrent que les élèves québécois de 13 et 16 ans obtiennent des résultats scolaires comparables ou supérieurs à la moyenne canadienne dans les matières scolaires scientifiques (Gouvernement du Québec, 2000a; 2000b), ils seraient moins nombreux à les apprécier (CST, 2002a; 2002b; CMEC, 2004). Ainsi, l'intérêt des élèves pour les études dans les champs scientifiques et technologiques diminuerait graduellement au cours du parcours scolaire (Désautels et Larochelle, 2004; Roth, 2006).

En ce sens, une des principales critiques pouvant être adressée à l'enseignement des ST renvoie à son immobilisme, à l'incapacité de cet enseignement de s'adapter et d'évoluer au rythme des avancées, tant scientifiques que pédagogiques (Barma et Guilbert, 2006; Paldy, 2003). Les auteurs déplorent la persistance d'un enseignement à prédominance traditionnelle, centré sur la transmission d'une « litanie de faits, empêchant plusieurs élèves de comprendre le rôle des scientifiques et de la communauté scientifique dans le cadre de l'activité scientifique » (Brown et al., 2006, p. 784-785; De Coito, 2006) et l'importance excessive donnée à la mémorisation (Brown et al., 2006; Tsai, 2001). Ils dénoncent aussi l'aspect factice des activités pratiques qui, dans leur état actuel, renforcent une pédagogie centrée sur l'enseignant (Hennessy et al., 2007; Suits, 2004). De même, ils déplorent le peu d'intérêt porté aux activités de débat ou de discussion, et ce, malgré l'importance que revêt l'aspect communicationnel pour le développement des connaissances scientifiques et technologiques (Haigh, France et Forret, 2005; Thouin, 2002).

Le constat ainsi posé soulève la question de l'adaptation de l'enseignement des sciences et technologies :

La diversité des enseignants et des contextes, l'hétérogénéité des élèves et la flexibilité de la discipline [technologie] laissent supposer une variété de mises en œuvre. Or, toutes les recherches sur les pratiques d'enseignement tendent à révéler, d'une part, leur relative uniformité et, d'autres part, la faible perception par les enseignants de l'hétérogénéité des publics et parmi les publics (Lebeaume, 2002, p. 66)

Ces recherches stigmatisent, pour la plupart, la résistance des démarches d'enseignement par rapport à toute tentative de changements (Amigues et Kherroubi, 2003), malgré les efforts des divers acteurs. 


\title{
La difficile adaptation des démarches d'enseignement
}

\author{
Selon Tardif (2005),
}

les pratiques pédagogiques des enseignants évoluent lentement parce que leur travail n'a guère varié dans ses dimensions fondamentales au fil du temps. [...] il est indéniable que l'enseignement, quand on le compare à la plupart des métiers et professions modernes, représente un travail que nous appellerons ici à évolution lente (p. 76).

Dans le domaine des ST, cette évolution serait d'autant plus difficile qu'elle se heurte également aux représentations des enseignants en lien avec la nature des contenus, l'enseignement et l'apprentissage des ST. Ces représentations renverraient le plus souvent à des perspectives réalistes (correspondance directe entre les objets et phénomènes et les éléments explicatifs proposés par la science) et inductives (faits scientifiques issus de l'observation) (Leach, Millar, Ryder et Séré, 2000). Ces représentations amèneraient les enseignants à considérer les savoirs scientifiques comme des faits préexistants incontestables, vérités absolues auxquelles ils accèdent pour les transmettre aux élèves, justifiant ainsi une préséance des modes d'enseignement traditionnels magistraux (Fensham, 2001). Aussi, la modification des démarches d'enseignement demeure-t-elle difficile (Gauthier, Gaudreau et Minier, 2007; Potvin, Fournier, Mauffette et Paradis, 2007).

Allant dans le même sens, les résultats d'une étude effectuée auprès d'enseignants de sciences d'expérience (Kang et Wallace, 2004) démontrent que plus les croyances entretenues sont complexes et s'éloignent des conceptions « naïves » (savoirs scientifiques neutres et cumulables), plus l'établissement d'un lien direct entre ces croyances et les pratiques adoptées en classe est ardu. Pour Buckmann et von Ossietzky (2005), ce lien peut s'avérer difficile à démontrer puisqu'il subirait l'influence d'une multitude de composantes (attitudes, intentions, contextes, temps, comportements, etc.) pouvant contrecarrer la mise en place de l'action projetée (Pourtois et Desmet, 2004). Il serait ainsi plus facile de mettre en place des pratiques transmissives que d'opter pour des pratiques favorisant des processus d'investigation représentatifs de l'activité scientifique.

Toutefois, en adoptant un point de vue «micro » (Tardif, 2005) centré sur la classe, il est possible de constater que l'homogénéité reconnue des démarches ne permet pas de faire ressortir les capacités de variation et d'adaptation des enseignants (Lebeaume, 2002); elle n'aide pas à comprendre l'habileté des enseignants à "improviser et s'adapter au contexte relativement fluide et changeant des interactions avec les élèves » (Tardif, 2005, p. 77). Il convient alors de se questionner sur les perspectives à adopter pour mieux rendre compte des processus d'adaptation pour éviter le piège de la répétition du discours, pour ouvrir sur une discussion riche et porteuse de changement.

\section{Vers la nécessaire étude des besoins}

Devant cette complexité, il devient nécessaire d'appréhender tant les aspects didactiques et épistémologiques de l'enseignement des ST, que les aspects pédagogiques, organisationnels ou environnementaux (Boutin, 2006). La notion de besoins permettrait cette ouverture sur la complexité des facteurs sous-tendant l'adaptation, puisqu'elle 
favoriserait l' « organisation structurée de l'information en matière de ressources et de méthodes pédagogiques [...] mais aussi une analyse critique des productions et des méthodes pédagogiques » (Gouvernement du Québec, 1997, p. 89). Elle serait d'autant plus pertinente puisque

les besoins poussent à l'action, car ils créent des tensions que la personne veut réduire ou éliminer. Le comportement motivationnel lié aux besoins peut être décrit de la façon suivante. L'apparition de besoins incite à vouloir les satisfaire, d'où des actions en conséquence (Louart, 2002, p. 3).

Ainsi, qu'il s'agisse des besoins de l'enseignant en lien avec son enseignement ou de ceux qu'il perçoit chez les élèves en lien avec leur apprentissage (CSE, 2004, 2006), ces besoins constitueraient des déclencheurs de l'intention d'action (Darpy, 2000).

L'enseignant serait alors le mieux placé pour évaluer les besoins en salle de classe et pour adapter l'enseignement en conséquence (Beck, Hart et Kosnik, 2002). En ce sens, l'étude des besoins pourrait se révéler un outil puissant « visant la mise au point de méthodes et de moyens nécessaires à l'enseignement et la formation [...] vers le réajustement de pratiques pédagogiques "dépassées" ou peu populaires » (Lapointe, 1992, p. 45).

Pourtant, comme le mentionne Lebeaume (2002), de telles études en enseignement des ST sont quasi-inexistantes. Le plus souvent, elles ne relèvent que sommairement quelques éléments de justification des démarches utilisées, de façon à informer des résultats centraux obtenus en termes de description des activités d'enseignement-apprentissage et des avantages qu'elles présentent sur le plan des apprentissages des élèves. Une étude approfondie de ces justifications et s'intéressant plus particulièrement aux besoins qui les sous-tendent permettrait d'accéder non seulement aux besoins de l'enseignant, mais également à ceux qu'il perçoit chez les élèves et qui peuvent l'amener à prendre des décisions adaptatives variées en cours d'action (Halai et Hodson, 2004). Il serait ainsi possible, en accord avec les recommandations de Kang et Wallace (2004), d'appréhender les situations d'enseignement des ST dans leur complexité et d'apporter un éclairage important par rapport au processus d'adaptation des démarches d'enseignement que vivent les enseignants de ST en contexte d'enseignement.

Ces constats soulèvent des questions, sur lesquelles repose la recherche présentée dans cet article. Considérant les spécificités des contextes d'enseignement et d'apprentissage des ST au secondaire, de même que la nature des concepts et des démarches scientifiques et technologiques :

1. comment le processus d'adaptation des démarches d'enseignement en cours d'action est-il influencé par les besoins déclarés des enseignants de ST de l'école secondaire?

2. comment le processus d'adaptation de ces démarches en cours d'action est-il influencé par les besoins perçus chez les élèves par les enseignants de ST de l'école secondaire?

3. quelles dynamiques d'action et d'adaptation de cet enseignement et de ses démarches peuvent alors être identifiées? 


\section{Méthodologie}

Treize enseignants ont participé à l'étude. L'échantillon était majoritairement composé de femmes, ayant dix années ou moins d'expérience d'enseignement. Au moment de la collecte de données, plusieurs des participants (8) enseignaient plus d'une matière.

Suivant la logique écosystémique selon laquelle les actions et les évolutions sont tributaires de la perception que l'individu se fait de son environnement (Bronfenbrenner, 1979, 1993), les adaptations apportées par l'enseignant aux démarches d'enseignement qu'il utilise en classe ne peuvent reposer que sur sa propre réalité, telle qu'il la perçoit et, par conséquent, sur les besoins perçus. Pour relever ces perceptions, quatre groupes de réflexion ont été effectués, chaque groupe comprenant trois personnes (à l'exception d'un groupe comprenant 4 personnes). Ce mode de collecte de données a été privilégié étant donné son efficacité lorsqu'il s'agit de recueillir des opinions ou des représentations et d'identifier ou clarifier des besoins (Fern, 2001; Krueger et Casey, 2000). Pour chaque groupe, le recueil des données a été effectué à l'aide du dispositif de Démarche Réflexive d'Analyse en Partenariat (DRAP) (Boudreault et Kalubi, 2006). Ce dispositif et le logiciel associé ont permis aux participants d'énoncer leurs idées, sans que celles-ci soit discutées ou jugées par les autres membres du groupe, ce qui réduit considérablement les effets d'entraînement ou de musellement pouvant se produire dans un groupe. De plus, la capture immédiate que permet le dispositif DRAP de chaque énoncé et sa projection in situ permet à l'émetteur de l'énoncé d'apporter des correctifs ou des nuances si nécessaire, et d'en valider le contenu auprès du chercheur (Ibid.). À l'issue de la collecte de données, un total de 678 énoncés avaient été recueillis.

Ces énoncés ont ensuite été soumis à une analyse de contenu (Bardin, 1998; 2003) de manière à effectuer un classement en deux temps. Dans un premier temps, il s'agissait d'identifier le «type » d'énoncé, de manière à permettre de « caractériser l'esprit de l'idée formulée » (Boudreault et Kalubi, 2006, p. 28). Il s'agit, en quelque sorte, de déterminer si l'énoncé est présenté selon un point de vue positif, négatif ou neutre. Dans le cadre de la présente recherche, la définition des types d'énoncés repose sur les théories de Darpy (2000) et de Purseigle (2004), en lien avec le processus décisionnel.

Ce cadrage théorique a permis d'identifier quatre types d'énoncés possibles, qui ont servi de base à l'établissement de la grille de classement :

- Les accélérateurs : il s'agit d'énoncés faisant état de besoins ressentis par les enseignants ou perçus par eux chez leurs élèves, qui les amènent à poser des actions allant dans le sens d'une adaptation de leur enseignement et des démarches mises en place;

- Les décélérateurs : ces énoncés identifient des obstacles à l'adaptation de l'enseignement ou des besoins pouvant amener l'enseignant à se retrancher sur les démarches habituellement utilisées;

- Les flottements : cette catégorie contient les énoncés pour lesquels il est difficile d'identifier clairement la position de l'enseignant, dans la mesure où l'élément énoncé peut constituer soit un accélérateur, soit un décélérateur selon les contextes, les caractéristiques des acteurs ou tout autre facteur variable dans une situation donnée; 
- Les éléments neutres : cette catégorie a permis la classification d'énoncés plus descriptifs, pour lesquels des liens avec l'adaptation des démarches d'enseignement ne pouvaient être établis.

Il est nécessaire de souligner ici un aspect qui n'apparaît pas, de prime abord, comme une évidence mais qu'il convient de garder à l'esprit : un accélérateur ne revêt pas toujours un aspect positif, pas plus qu'un décélérateur se décline nécessairement de manière négative. Ainsi, des difficultés en raison des comportements des élèves, par exemple, peuvent représenter un accélérateur puissant et obliger l'enseignant à adapter rapidement la démarche d'enseignement pour réduire les écarts de conduite. À l'opposé, l'expression par les élèves de leur intérêt, événement positif s'il en est, peut devenir un décélérateur puisque, les élèves étant intéressés, l'enseignant ne ressentira pas le besoin d'adapter la démarche utilisée.

Dans un deuxième temps, des « attributs » ont été alloués aux énoncés recueillis, de manière à préciser davantage leur caractère (Boudreault et Kalubi, 2006). La recherche s'attachant à comprendre le processus d'adaptation des démarches d'enseignement sur la base des besoins perçus par les enseignants, la classification élaborée s'inspire du modèle des douze besoins de Pourtois et Desmet $(1997,2004)$ structuré autour de quatre dimensions principales :

- Les besoins d'ordre affectif sont des arguments liés à la motivation et au développement de l'intérêt, au plaisir relié au travail ou aux tâches à effectuer, aux attitudes des acteurs, aux sentiments d'appartenance, de sécurité et de reconnaissance;

- Les besoins d'ordre cognitif regroupent des considérations de l'ordre du potentiel de réussite, de la formation et des apprentissages, du contrôle et de l'aisance par rapport aux savoirs, ainsi que de la construction de sens par les acteurs;

- Les besoins d'ordre social ou relationnel renvoient à l'espace communicationnel entre les acteurs, aux interactions et aux relations qui s'y tissent, de même qu'à l'établissement de réseau de soutien;

- Les besoins d'ordre idéologique s'intéressent à la transmission de valeurs collectives, d'éléments de culture et d'idéaux, ainsi qu'aux perspectives épistémologiques adoptées par les acteurs.

Puisque le contexte de base du développement du modèle des douze besoins réfère au développement de l'enfant et des liens familiaux, il a été nécessaire de recourir à d'autres études effectuées en contexte scolaire afin de compléter la catégorisation, de manière à intégrer des éléments spécifiques au contexte scolaire. En consultant plusieurs études, il a été possible d'ajouter deux dimensions:

- Les besoins d'ordre organisationnel renvoient aux aspects relatifs à la gestion de la classe et au contrôle des comportements, à l'organisation du temps et de l'espace (Hodson, 2006; Richoux et Beaufils, 2005);

- Les besoins d'ordre structurel regroupent les éléments liés au curriculum, à l'établissement, à la culture scolaire et à l'environnement scolaire (Vérin, 1998), de même que les enjeux de l'ordre des ressources humaines et techniques (Hennessy et al., 2007). 
Afin d'assurer la validité des grilles de classement (types et attributs), les catégories définies ont été soumises à l'examen d'experts. Toutefois, malgré le soin porté à la définition et la précision des catégories, il est rare que les entités à regrouper présentent les attributs d'une seule d'entre elles (Huberman et Miles, 1991), ce qui complique considérablement la tâche de l'analyste et pose le danger d'une classification arbitraire. Cette nature souvent équivoque des énoncés oblige le chercheur à définir clairement les indicateurs relatifs à chaque catégorie, ainsi qu'à identifier l'idée principale ou directrice pour chaque unité à classer (De Landsheere, 1982). Afin de s'assurer de la fiabilité des catégories ainsi construites, la classification des énoncés a été effectuée à l'aide d'une procédure d'accord interjuges. Cette procédure a fait appel à deux juges (la chercheure et une personne externe), qui ont classé chaque énoncé dans l'une ou l'autre des catégories composant les grilles de lecture. À la suite d'une première classification sur près d'une centaine d'énoncés, le coefficient kappa (k) de Cohen (Janson et Olsson, 2004; Watkins et Pacheco, 2001) a été calculé pour chaque classification utilisée. Le coefficient kappa de Cohen est un coefficient de corrélation s'étalant entre 0 (aucun accord) et 1 (accord parfait). Il a la particularité de s'appliquer spécifiquement au cas où deux juges sont utilisés, pour le classement en fonction de variables nominales. Une valeur élevée du coefficient kappa signifie qu'il n'est pas nécessaire d'avoir recours à un plus grand nombre de juges puisque les catégories et le classement des juges dans ces catégories sont fiables (Begsten et Piérard, 2006).

Contrairement au pourcentage d'accord, le coefficient kappa de Cohen admet un ajustement permettant de soustraire les accords pouvant être attribués au hasard. Pour les énoncés où il y avait désaccord entre les deux juges, une tierce personne a été mise à contribution afin de décider de la catégorie finale de l'énoncé.

À l'issue de cette opération, les coefficients Kappa de Cohen (k) obtenus pour chacune des grilles étaient satisfaisants ${ }^{1}$; les degrés d'accord interjuges obtenus pour les types d'énoncés et les attributs représentaient tous deux un bon degré de fiabilité, soit 0,6441 et 0,7135 respectivement.

\section{Résultats}

La répartition des énoncés recueillis montre que les accélérateurs se présentent en nombre plus important (44\%) que les décélérateurs (22\%). Conformément aux propos de Monfroy (2002), l'adaptation ferait donc partie intégrante du quotidien des enseignants. Les accélérateurs renvoient surtout aux besoins d'ordre cognitif. Quant aux décélérateurs, ils se répartissent essentiellement dans les catégories des besoins cognitifs, structurels et organisationnels, avec une prédominance des besoins cognitifs (figure 1). Ainsi, alors que l'adaptation des démarches d'enseignement en ST est le plus souvent soutenue par des préoccupations relatives à l'apprentissage, à l'enseignement et aux savoirs scientifiques et technologiques, ce serait non seulement ces préoccupations, mais également celles en lien avec la gestion de l'enseignement (gestion du temps, des comportements, de l'hétérogénéité; structuration des activités), les ressources humaines et matérielles, le curriculum d'enseignement des ST, la culture scolaire ou des contraintes relevant de l'établissement scolaire qui viendraient freiner l'adaptation des démarches.

\footnotetext{
${ }^{1}$ Selon Watkins et Pacheco (2001), les valeurs du coefficient kappa de Cohen peuvent être interprétées comme suit : moins de $0,40=$ degré d'accord pauvre; entre 0,40 et $0,60=$ degré d'accord acceptable; entre 0,60 et $0,75=$ bon degré d'accord; plus de $0,75=$ degré d'accord excellent.
} 
Figure 1

Répartition des énoncés selon les catégories et les types

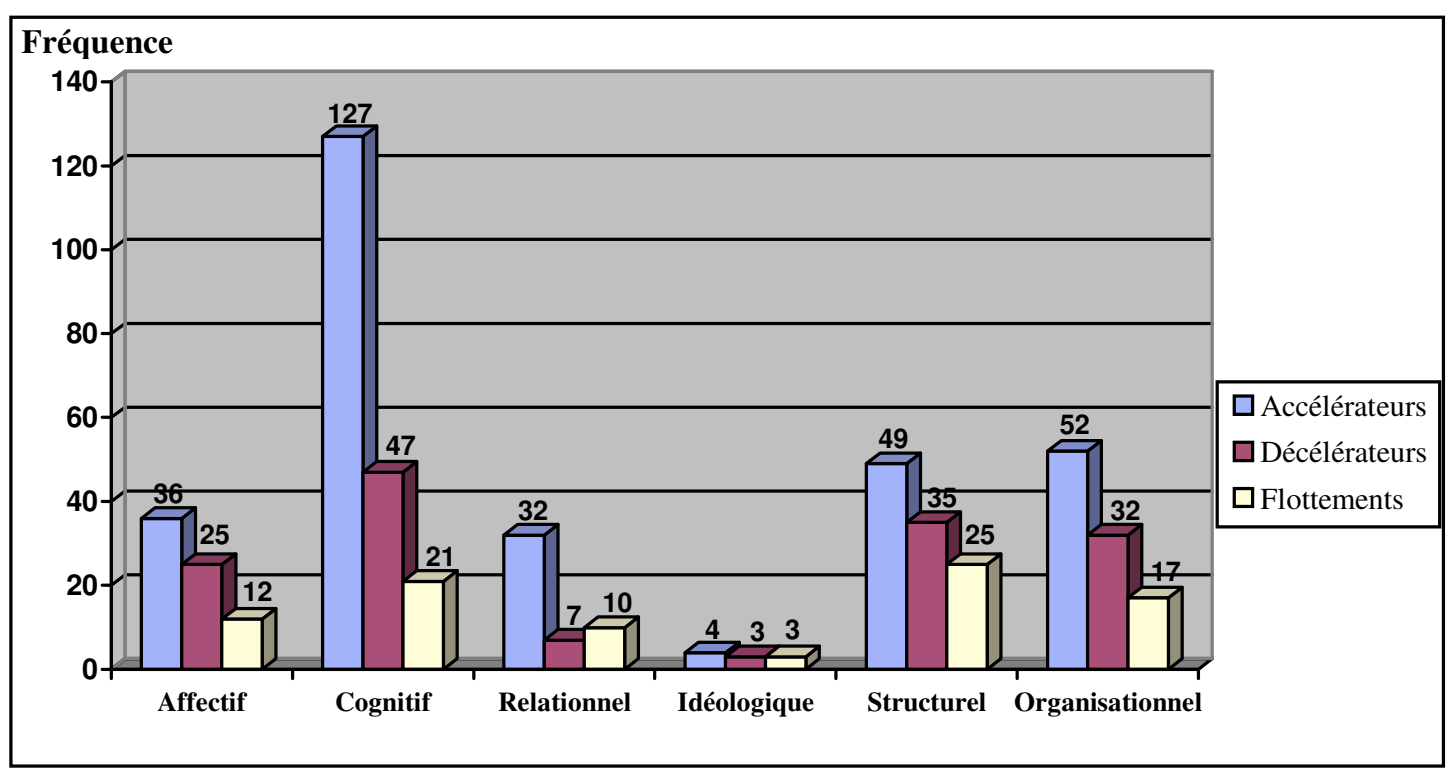

Il est à noter que certains énoncés n'ont pu être identifiés en tant qu'accélérateurs ou décélérateurs (flottement, 13\%), les enseignants ne s'étant pas clairement positionnés lors de l'énonciation. Afin de limiter l'apport subjectif des chercheurs, ces énoncés n'ont pas été reclassés dans l'une ou l'autre catégorie et ne seront pas pris en compte. De même, les éléments neutres ( $21 \%$ ) ne seront pas considérés puisqu'ils n'apportent aucune information sur l'adaptation des démarches d'enseignement.

De manière plus spécifique, les accélérateurs peuvent être présentés selon deux orientations différentes. En effet, certains accélérateurs sont identifiés comme éléments stimulant ou obligeant l'adaptation de l'enseignement des ST, tandis que d'autres sont considérés comme des formes de soutien ou des facilitateurs de cette adaptation.

Parmi les facteurs obligeant les enseignants de ST à adapter leur enseignement, on retrouve plusieurs énoncés qui renvoient aux élèves, à leur développement et à leur apprentissage des concepts scientifiques et technologiques. De fait, plusieurs des énoncés relevés font état de l'obligation d'adaptation continuelle que posent la diversité des goûts et des intérêts des élèves, des niveaux académiques, des profils d'élèves (difficultés spécifiques, retard scolaire, comportements, etc.) et de groupes (groupe plus ou moins difficile), des rythmes d'évolution dans l'apprentissage et les degrés d'autonomie atteints en cours d'année. Les enseignants seraient alors contraints d'adapter le degré de difficulté des activités de façon à présenter aux élèves des défis ni trop faciles à relever, ni trop ardus. En ce sens, ils seraient attentifs aux indices permettant d'identifier les incompréhensions et les difficultés des élèves, de manière à pouvoir y remédier le plus rapidement possible : «si j'ai l'impression que les élèves sont totalement perdus et qu'il n'y a pas de moyen pour les "déperdre" je vais modifier mon approche ». La nécessité de soutenir l'apprentissage de l'élève sous-tend toutefois un second besoin, cette fois chez l'enseignant de ST. Pour ce dernier, la qualité de l'apprentissage de l'élève serait un indicateur de son degré de compétence. Il éprouverait alors le besoin de constater que les 
actions entreprises sont efficaces sur les plans de l'apprentissage, du développement et de l'évolution des élèves : «j'ai besoin de sentir que ce que je fais donne des résultats ».

D'autres facteurs stimulant l'adaptation des démarches d'enseignement par les enseignants de ST sont à mettre en lien avec les contextes d'enseignement. Parmi eux, notons le moment de l'année (avant ou après des vacances, début ou fin d'année, etc.) ou de la journée (première ou dernière période, après un cours d'éducation physique, etc.) où se produit le cours de ST. La gestion du temps d'enseignement imposerait également des contraintes importantes, particulièrement en contexte de laboratoire ou d'atelier pratique : «Parfois, je me rends compte que l'activité ou le laboratoire prend plus de temps que ce que j'avais prévu; je dois donc adapter l'enseignement rapidement ». De même, les objectifs fixés pour une leçon, les notions et concepts abordées et les compétences à développer amèneraient les enseignants de ST à varier leurs démarches d'enseignement, de façon à obtenir la combinaison la plus efficace possible : «je fais de l'enseignement magistral pour clarifier des concepts que les élèves ont de la difficulté à saisir dans les projets ».

Enfin, des facteurs externes (hors école) motiveraient l'adaptation des démarches d'enseignement. Une adaptation serait à effectuer en fonction de la culture locale, du milieu d'appartenance, de façon à rendre l'enseignement des ST le plus significatif possible : «si je change d'école ou de milieu, je dois m'adapter avec la région dans laquelle je suis ». Toutefois, l'influence externe la plus importante proviendrait de l'application de la réforme présentement en cours au Québec et des modifications importantes qu'elle provoque, particulièrement pour les enseignants de ST. Face à cette nouvelle réalité (regroupement des matières, enseignement à toutes les années du secondaire, intégration de compétences, etc.), les enseignants se verraient poussés à une adaptation rapide pour répondre aux exigences en termes de démarches d'enseignement préconisées ( «La réforme a changé la façon d'aborder la matière »), mais également en termes de structuration et de planification des contenus, étant donné l'introduction de l'enseignement par cycle : « les enseignantes qui enseignent en sciences sont dans les deux premières années de la réforme; elles vont s'adapter pour les prochaines années et s'ajuster ».

Du côté des facteurs soutenant l'adaptation, les énoncés relatifs aux relations entre enseignants, au soutien de la direction et à la disponibilité des ressources humaines et matérielles sont prédominants. Ainsi, les échanges entre enseignants de ST représenteraient une source stimulante d'idées et de connaissances vers l'essai de nouvelles activités d'enseignement-apprentissage ou de nouvelles pratiques : «c'est important de partager nos expériences et nos connaissances entre enseignants »; «ça me stimule énormément d'être avec quelqu'un, de partager des idées ». Quant au soutien de la direction, il donnerait de l'assurance aux enseignants et favoriserait le développement du sentiment de compétence, lesquels auraient des effets positifs sur l'enseignement et la confiance déployée lors de l'utilisation de démarches d'enseignement alternatives. Enfin, les effets de la disponibilité des ressources humaines et matérielles seraient duels : d'une part, leur accessibilité permettrait aux enseignants de ST d'adapter plus facilement leur enseignement : «Avoir plus de ressources (matérielles et humaines), de budget, d'outils, etc. permettrait d'améliorer mon enseignement ». D'autre part, leur dysfonctionnement (ressources matérielles) ou leur absence forcerait à trouver des solutions de rechange : 
«si les ressources matérielles ne sont pas disponibles pour une activité, il faut faire autrement et s'adapter ».

Toutefois, la problématique des ressources matérielles et humaines renvoie également à la catégorie des décélérateurs. Se retrouvent également dans cette catégorie l'inadéquation des formations initiale et continue en lien avec la réforme, les lacunes associées à cette dernière, les difficultés des élèves et la variabilité de leur profil au sein d'un même groupe, les réactions positives des élèves et la résistance des enseignants envers le travail d'équipe (entre collègues).

En ce qui concerne les ressources humaines et matérielles, elles pourraient être limitatrices, dans la mesure où elles ne permettraient pas de répondre aux besoins des élèves et des enseignants de ST en termes de soutien : " je ne peux pas tester des activités dans les groupes plus difficiles même si je sais que certains élèves fonctionneraient mieux, parce que je n'ai pas le soutien nécessaire (intervenant, orthopédagogue, etc.) ». Quant aux besoins de formation, ils ne seraient pas totalement comblés, ce qui rendrait difficile l'appropriation de la réforme et des modes d'enseignement qu'elle préconise : «j'essaie de m'approprier les éléments de clarification par rapport aux approches vues dans la formation mais ce n'est pas toujours facile »; « les formations ne sont pas toujours pertinentes pour mettre la réforme en pratique ». De même, la formation (initiale et continue) reçue par les enseignants de ST serait parfois incomplète et ne permettrait que partiellement de faire face aux réalités de l'enseignement : " on a beau voir toutes les belles théories sur la gestion des comportements dans la formation, tant qu'on ne les vit pas ça ne peut pas être pratique ». L'intégration du champ de la technologie et le décloisonnement des matières scientifiques stimuleraient également les besoins de formation : «il faudrait améliorer la formation sur la technologie »; « il faudrait réacquérir certaines connaissances; des notions avec lesquelles on est moins à l'aise ».

L'évaluation dans le cadre de la réforme constituerait aussi un problème important : «les grilles d'évaluation ne permettent pas d'évaluer tout le monde au cours d'une même activité parce qu'elles sont trop lourdes (longues) ». De même, la pression mise sur les enseignants de ST serait considérable, particulièrement en raison de la lourdeur du programme de ST et d'une tension perçue entre le développement des compétences et celle des contenus scientifiques et technologiques : «je me sens tiraillée entre le souci de faire ce qu'on me demande (réforme) et ce que je voudrais faire et qui me permettrait de me respecter dans ce que je suis »; « le contenu est plus important (plus de notions) qu'avant, le temps pour l'apprendre aussi, mais on a le même temps d'enseignement ». Enfin, les difficultés d'apprentissage et de comportement des élèves rendraient certaines démarches difficiles à appliquer : «ça prend beaucoup plus de temps à réaliser un projet avec un groupe difficile qu'avec un groupe qui fonctionne mieux sur le plan du comportement ». Ces différentes lacunes provoqueraient de l'insécurité, freinant l'adaptation de l'enseignement des ST.

D'autres décélérateurs sont à mettre en lien avec les caractéristiques des élèves. Ainsi, les enseignants de ST relèvent les difficultés de certains élèves à travailler en équipe ou en coopération, étant donné des habiletés sociales parfois déficientes. Pour eux, ces difficultés constitueraient un obstacle de taille étant donné que la nature des activités scientifiques et technologiques requerrait fréquemment un travail en collaboration de la part des élèves. Cela provoquerait des problèmes de gestion additionnels pouvant être décourageants pour les enseignants : «c'est très difficile; il faut que tu éteignes des feux, 
il y a des chicanes, tu ne peux pas tout voir (enseignement coopératif, évaluation) »; «j'ai beau essayer d'adapter mes méthodes et mes techniques, dans certains groupes difficiles, je ne peux même pas le faire parce que tout ce que je fais c'est de l'éducation en classe ». La composition des groupes représenterait alors une variable importante, tant sur le plan du nombre d'élèves que sur le plan de l'écart dans les niveaux : «c'est à la direction de gérer la distribution des élèves en difficulté et de réduire les groupes en conséquence, pour pouvoir avoir un niveau d'enseignement adéquat et adapté à leurs besoins ».

D'un autre côté, les réactions positives des élèves peuvent aussi provoquer une décélération sur le plan de l'adaptation de l'enseignement. De fait, si les élèves se montrent intéressés et si l'apprentissage effectué est satisfaisant, les enseignants n'auraient aucune raison de modifier la démarche d'enseignement utilisée: « je conserve certaines démarches quand je vois que les élèves réussissent bien sur le plan académique »; « ce n'est pas juste si les élèves ont aimé ça qui m'amène à conserver une situation d'apprentissage; c'est aussi leur motivation, leur engagement dans la tâche ».

Finalement, la réticence de certains collègues à travailler en collaboration constituerait un autre problème important. Certains des enseignants rencontrés vont même jusqu'à dénoncer la présence d'une culture enseignante fondamentalement individualiste, qui limiterait les échanges et les possibilités de partage constructif : «j'ai rarement retrouvé dans un milieu des profs qui veulent vraiment travailler en équipe ».

\section{Discussion}

Le métier d'enseignant est d'une grande complexité (Morandi, 2006; Tardif, 2006; Lessard, 2005). Il nécessite la prise en compte d'une «infinité de facteurs agissant en synergie » (Baby, 2005, p. 185), qui doivent être pris en compte lorsqu'il est question d'adaptation (ou d'inadaptation) au sein du système scolaire. Les données recueillies font largement état de cette complexité. Elles mettent en évidence une grande variété de besoins perçus par les enseignants de ST, de même que la versatilité de ces besoins (tantôt obstacles, tantôt stimulants) selon les interprétations, les contextes ou les conditions ponctuelles dans lesquelles se déroule l'action. Dès lors, il ne saurait être question d'un processus d'adaptation des démarches d'enseignement. Il faudrait plutôt aborder cette adaptation comme un effort visant à répondre à des besoins multiples. Conséquemment, cet effort d'adaptation peut prendre plusieurs formes, résultant en la création de processus d'adaptation multiples.

Les résultats obtenus montrent la prédominance des besoins de type cognitif dans le discours des enseignants de ST et qu'ils sont la plus grande source d'accélération. Cela indique que, la plupart du temps, adaptation rime avec apprentissage. De manière plus explicite, cela signifie que l'adaptation des démarches d'enseignement en ST se fait le plus souvent pour favoriser l'apprentissage de l'élève (Bourassa, Serre et Ross, 2000; Desrosiers et Malo, 2004). Dans le cas présent, ces apprentissages renvoient le plus souvent à la compréhension des concepts scientifiques et technologiques, ainsi que des démarches reliées à ces disciplines. L'adaptation est donc résolument tournée vers l'élève, ce qui n'a rien de surprenant dans un système sensé l'instruire, le socialiser et l'éduquer.

Les besoins cognitifs attribués aux élèves s'expriment dans l'action de façon ponctuelle. Ils amènent l'enseignant de ST à se demander si l'élève est intéressé par ce 
qu'il fait actuellement, s'il est disponible pour apprendre et intégrer les concepts à l'étude, s'il éprouve des difficultés particulières par rapport à la notion enseignée; bref, s'il apprend suffisamment pour le temps et les efforts déployés. Ainsi, la complexité des concepts et notions scientifiques et technologiques, de même que les particularités liées aux critères de scientificité des démarches et méthodes de travail en ST sont à mettre étroitement en lien avec l'adaptation des démarches d'enseignement. Les besoins des élèves sur ces plans forcent les enseignants de ST à agir, à réajuster en cours d'action, à effectuer des adaptations constantes. Comme l'indiquent Yvon et Garon (2006), ce processus itératif d'actions/réactions serait à la source d'innovations : «Dans le cas où cette forme [d'enseignement] est mise à mal par l'activité des élèves, les enseignants n'ont d'autres choix que d'innover en puisant dans leurs ressources propres les moyens de faire face à cette difficulté » (p. 74).

Il ne faudrait toutefois pas prétendre que toute adaptation aux besoins des élèves s'effectue dans l'action. En effet, les résultats obtenus présentent également l'adaptation en termes de prévision des difficultés des élèves et d'adaptation des contenus et de l'enseignement effectuées a priori, lors de la planification. C'est ce que certains auteurs nomment le pedagogical content knowledge (Halai et Hodson, 2004; Malo, 2000). Cette adaptation a priori fait intervenir d'autres besoins, cette fois chez les enseignants. Ces besoins renvoient surtout aux mesures de soutien à l'enseignement, aux conditions nécessaires pour rendre cet enseignement le plus efficace possible. Dans le cadre de l'enseignement des ST, ces besoins s'expriment surtout en lien avec le contexte d'enseignement en laboratoire. Il s'agit alors de s'assurer de la disponibilité des locaux, des techniciens et des spécialistes, dans la mesure où les activités d'expérimentation nécessitent une préparation particulière en termes de matériel (disponibilité, montage, fonctionnement) et de soutien à l'élève. Les besoins des enseignants sont généralement à prendre en compte lors de la planification : la satisfaction ou non de ces besoins est prévisible et donne lieu à des choix réfléchis, à des adaptations avant l'action.

Les adaptations en cours d'action constitueraient un deuxième niveau adaptatif. Elles se produiraient lorsque les premières adaptations effectuées lors de la planification s'avèrent insuffisantes ou inefficaces ou lorsque, au-delà de caractéristiques communes à tous les élèves, l'hétérogénéité sur les plans des besoins, des intérêts, des habiletés, des niveaux d'expérience, de la disponibilité et de l'origine socioculturelle (DeCoito, 2006) rend difficile l'identification a priori des adaptations nécessaires.

Les besoins cognitifs ne constituent cependant pas uniquement une incitation à l'adaptation : ils possèdent un potentiel inhibiteur important, lorsque l'hétérogénéité des profils des élèves est trop grande ou lorsque les difficultés ciblées dépassent le champ d'expertise de l'enseignant. Il arriverait donc parfois que l'activité des élèves amène plutôt les enseignants de ST à éviter les innovations et à se retrancher sur du connu qui, chez ces enseignants, prend le plus souvent la forme d'enneigements magistraux et d'exercisation. Qu'il s'agisse des besoins attribués à l'élève ou de ceux ressentis par l'enseignant, une même conclusion s'impose : l'adaptation de l'enseignement s'exprime d'une manière duelle. Si les besoins perçus peuvent représenter une source de stimulation puissante vers l'essai de nouvelles démarches d'enseignement ou l'adaptation de démarches déjà utilisées, ces mêmes besoins peuvent aussi freiner cette adaptation, la retrancher dans ses campements. 
Ce constat soulève la question suivante : à quelles conditions ou dans quelles conditions les besoins identifiés deviennent-ils des stimulants plutôt que des inhibiteurs d'adaptation et d'innovation? Pour Barma et Guilbert (2006), il s'agirait d'une équation coûts-bénéfices reposant initialement sur le besoin de résoudre une insatisfaction. L'enseignant doit concevoir que son action aura les effets attendus ou qu'elle vaut la peine d'être tentée. Comme l'indiquent Halai et Hodson (2004), tout dépendrait de l'enseignant, de ses expériences en classe et hors classe, des besoins perçus chez les élèves, des contextes et des situations. Cela revient à dire que des liens étroits existent et se tissent continuellement entre l'expérience d'enseignement et l'expérience d'apprentissage (Morandi, 2006), que l'activité enseignante, que ses actions et ses résultats, ne peuvent être mieux compris que dans leur contexte (Roth, 2005).

La centration du discours sur les besoins cognitifs permet également de soulever un second point : celui de la relative importance accordée par les enseignants de ST aux difficultés de comportement. Même si plusieurs écrits font largement état de la préoccupation des enseignants par rapport à l'aspect de gestion de la classe (Furlán, 1998; Legault, 1993, Nault et Fijalkow, 1999), les résultats présentés montrent que les besoins organisationnels (stimulés en partie par des difficultés liées aux comportements et à la gestion de la classe) des enseignants de ST ne sont pas prédominants dans le discours analysé. Qui plus est, les comportements dérangeants adoptés par les élèves seraient surtout identifiés par ces enseignants comme étant des signes ou des indices que les besoins cognitifs (compréhension, intégration des apprentissages et concepts scientifiques) et affectifs (intérêt envers les sujets traités et motivation) ne sont pas comblés. Les enseignants de ST seraient donc davantage centrés sur l'apprentissage que sur les comportements. Ils répondraient de manière professionnelle en faisant preuve de compétence au sens entendu par le MELS, qui souligne que l'enseignant doit faire preuve $\mathrm{du}$ « discernement qui oriente l'attention du maître vers les indices d'un déséquilibre [...], indices qui l'avisent d'enclencher et d'aménager des démarches d'apprentissage pour créer de nouveaux équilibres » (Gouvernement du Québec, 2004, p. 85). Il serait alors pertinent d'aborder sous un angle différent l'étude des difficultés de comportements des élèves : plutôt que de voir ces difficultés comme la cause de tous les maux des enseignants, peut-être serait-il plus éclairant de les considérer, à l'instar des enseignants de ST ayant participé à l'étude, comme des indices de problèmes se situant à d'autres niveaux (cognitif ou affectif, par exemple) et pouvant orienter la recherche vers les causes de ces comportements inadaptés.

\section{Conclusion}

L'adaptation des démarches d'enseignement est un processus complexe. Les résultats de la recherche font état de la multiplicité des parcours d'adaptation possibles, par la grande richesse, la variabilité et les nuances retrouvées dans le discours analysé. Trois invariants demeurent cependant : 1) l'adaptation est une réponse à l'insatisfaction (Brassard, 2005); 2) l'adaptation doit être soutenue de manière adéquate sans quoi elle risque fort d'être compromise (Baby, 2005; Pelletier, 2005); 3) l'adaptation résulte d'une responsabilité entre l'enseignant et l'élève (Ménard et Laferrière, 2006; Morandi, 2006). 
Même si elle demeure incomplète en raison de son caractère exploratoire, cette analyse des besoins des enseignants de ST a permis de mettre en évidence l'importance de considérer ces besoins pour espérer faciliter l'adaptation des démarches d'enseignement. Elle souligne l'implication et le professionnalisme de l'enseignant de ST dans sa volonté de promouvoir un enseignement adapté aux besoins des élèves, quelles que soient les difficultés que posent ces besoins et que rencontrent les enseignants. La recherche démontre également la richesse de l'approche centrée sur les besoins en tant que source d'informations sur les réalités de l'enseignement des ST. À ce titre, cette recherche est porteuse d'innovation et offre des nouvelles perspectives de recherche, dans une optique de construction de voies d'action et de soutien qui répondent aux impératifs des changements sociaux actuels, tout en mobilisant les enseignants.

Même si nos résultats apportent des éléments de compréhension additionnels en ce qui concerne le processus d'adaptation des démarches d'enseignement en ST et les diverses influences qu'exercent les besoins relevés, ils ne peuvent répondre entièrement à la question de l'articulation (ou du point d'inflexion) entre accélérateurs et décélérateurs, entre stimulation et inhibition des adaptations. Des recherches plus avant, mettant en relation l'observation de l'enseignement des ST en contextes spécifiques (notamment en laboratoire ou en atelier) de même que l'explicitation par les enseignants des adaptations effectuées et des justifications qui les sous-tendent, seraient susceptibles d'apporter des éléments de réponses additionnels. De même, il serait fort intéressant d'explorer les perceptions des élèves des cours de ST par rapport à l'adaptation des démarches d'enseignement effectuées par leurs enseignants, puisqu'il est justifié de penser que les perceptions des enseignants et des élèves à ce sujet ne soient pas parfaitement arrimées (Lang, 2005; Leloup, 2003). De telles recherches permettraient de mieux comprendre les perceptions que se font les élèves de l'enseignement des ST ainsi que de l'adéquation des processus d'adaptation des démarches d'enseignement employés par leurs enseignants et pourraient apporter des indices quant aux adaptations les plus efficaces pour favoriser la motivation, l'engagement, l'intégration des savoirs scientifiques et technologiques et, ultimement, la réussite des élèves dans cette discipline. 


\section{Références}

Amigues, R. et Kherroubi, M. (2003). Éditorial. Recherche et formation pour les professions de l'éducation, 44, 5-10.

Baby, A. (2005). La pédagogie des poqués. Sainte-Foy: Presses de l'Université du Québec.

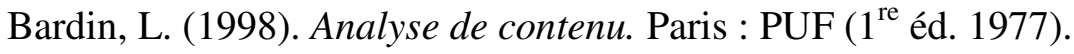

Bardin, L. (2003). L'analyse de contenu et de la forme des communications. In S. Moscovici et F. Buscini (dir.), Les méthodes des sciences humaines (p. 243-270). Paris : Presses universitaires de France.

Barma, S. et Guilbert, L. (2006). Différentes visions de la culture scientifique et technologique. Des défis et contraintes pour les enseignants. In A. Hasni, Y. Lenoir et J. Lebeaume (dir.), La formation à l'enseignement des sciences et des technologies au secondaire dans le contexte des réformes par compétences (p. 1139). Québec : Presses de l'Université du Québec.

Beck, C., Hart, D. et Kosnik, C. (2002). The teaching standards movement and current teaching practices. Canadian Journal of Education, 27(2-3), 175-194.

Boudreault, P. et Kalubi, J.-C. (2006). Animation de groupes. Une démarche réflexive d'analyse. Outremont : Carte Blanche.

Bourassa, B., Serre, F. et Ross, D. (2000). Apprendre de son expérience. Sainte-Foy: Presses de l'Université du Québec.

Boutin, G. (2006). L'école face à ses nouveaux défis. In Y. Montoya, J.-P. Martinez et G. Boutin (dir.), L'école actuelle face au changement. Instruire, éduquer ou socialiser (p. 3-16). Québec : Presses de l'Université du Québec.

Brassard, A. (2005). La gestion de la transformation : regard sur l'expérience québécoise de réforme curriculaire. In D. Biron, M. Cividini et J.-F. Desbiens (dir.), $L a$ profession enseignante au temps des réformes (p. 103-122). Sherbrooke : Éditions du CRP.

Bronfenbrenner, U. (1979). The ecology of human development. Cambridge, MA: Harvard University Press.

Bronfenbrenner, U. (1993). The ecology of cognitive development: Research models and fugitive findings. In R. Wozniak et K. W. Fischer (dir.), Development in context (p. 3-44). Hillsdale, NJ: Lawrence Erlbaum.

Brock Education, Volume 19, No. 1, Fall 2009, 73-93 
Brown, P.L., Abell, S.K., Demir, A. et Schmidt, F.J. (2006). College science teachers' views of classroom inquiry. Science Education, 90, 748-802.

Buckmann, C. et von Ossietzky, C. (2005). Young teachers' implicit theories about individual factors of school achievement. Document téléaccessible à l'URL $<$ http:// www.see-educoop. net/education_in/pdf/erasmus2005-oth-enl-t03.pdf> (Consulté le 10 juin 2005).

Conseil de la science et de la technologie (CST) (2002a). La culture scientifique et technique au Québec : bilan. Québec : Gouvernement du Québec.

Conseil de la science et de la technologie (CST) (2002b). Enquête sur la culture scientifique et technique des Québécoises et des Québécois. Québec: Gouvernement du Québec.

Conseil des Ministres de l'Éducation du Canada (CMEC) (2004). Programme d'indicateurs de la réussite scolaire PIRS Sciences III. Toronto: Conseil des Ministres (Canada).

Conseil supérieur de l'éducation (CSE) (2004). Un nouveau souffle pour la profession enseignante. Avis au ministre. Québec : Gouvernement du Québec.

Darpy, D. (2000). Importance de la procrastination dans le processus d'achat. Approche sémiotique et mesure. Communication présentée dans le cadre du $16^{\mathrm{e}}$ congrès de l'Association française de marketing (AFM), Montréal, Canada. Document téléaccessible à l'adresse <http://perso.wanadoo.fr/denis.darpy/Recherche/ AFM_2000.pdf>. (Consulté le 9 janvier 2006.)

DeCoito, I. (2006). Innovation in science education: challenging and changing teachers' roles and beliefs. Revue canadienne de l'enseignement des sciences, des mathématiques et des technologies, 6(4), 339-350.

Désautels, J. et Larochelle, M. (2004). Forme scolaire, éducation aux sciences et pratique de la critique. Revue canadienne de l'enseignement des sciences, des mathématiques et des technologies, 4(4), 515-528.

Desrosiers, P. et Malo, A. (2004). Démarche de réflexion partagée entre formateurs de terrain et stagiaires. Communication présentée dans le cadre de la $7^{\mathrm{e}}$ Biennale de l'éducation et de la formation, 14-17 avril, Lyon, France. Document téléaccessible à l'adresse <www.inrp.fr/Access/Biennale/7biennale/Contib/longue/7123.pdf>. (Consulté le 17 août 2007.)

Fensham, P.J. (2001). Science content as problematic. In H. Behrendt et al. (dir.), Research in science education - Past, present, and future (p. 27-41). Dordrecht: Kluwer Academic Publishers.

Fern, E.F. (2001). Advanced focus group research. Thousand Oaks, CA: Sage Publications. 
Furlán, A. (1998). Problèmes de discipline dans les écoles du Mexique : le silence de la pédagogie. Perspectives, XXVIII(4), 611-627.

Gauthier, D., Gaudreau, D. et Minier, P. (2007). La modification des pratiques enseignantes en sciences et technologies. Communication présentée dans le cadre du $75^{\mathrm{e}}$ congrès de l'ACFAS, Trois-Rivières, Canada, 7-11 mai.

Giordan, A. (1999). Une didactique pour les sciences expérimentales. Paris : Belin.

Gouvernement du Québec (1997). Réaffirmer l'école. Rapport du groupe de travail sur la réforme du curriculum. Québec : Ministère de l'Éducation.

Gouvernement du Québec (2000a). Les résultats - L'évaluation des apprentissages. L'apprentissage des sciences chez les élèves de 13 ans. Québec: Ministère de l'Éducation. Document téléaccessible à l'adresse <http://www.meq.gouv.qc.ca/ stat/indic00/indic00f/vf00404.pdf>.(Consulté le 19 août 2007)

Gouvernement du Québec (2000b). Les résultats.- L'évaluation des apprentissages. L'apprentissage des sciences chez les élèves de 16 ans. Québec: Ministère de l'Éducation. Document téléaccessible à l'adresse <http://www.meq.gouv.qc.ca/ stat/indic00/indic00f/vf00405.pdf >. (Consulté le 19 août 2007)

Gouvernement du Québec (2004). La formation à l'enseignement. Les orientations. Les compétences professionnelles. Québec : Ministère de l'Éducation.

Haigh, M., France, B. et Forret, M. (2005). Is 'doing science' in New Zealand an expression of scientific inquiry? International Journal of Science Education, 27(2), 215-226.

Halai, N. et Hodson, D. (2004). Munazza's story: shedding light on a science teacher's conceptions of the nature of science through a life history study. Revue canadienne de l'enseignement des sciences, des mathématiques et des technologies, 4(2), 193208.

Hennessy, S., Wishart, J., Whitelock, D., Deany, R., Brawn, R., la Velle, L., McFarlane, A., Ruthen, K et Winterbottom, M. (2007). Pedagogical approaches for technologyintegrated science teaching. Computers \& Education,48(1), 137-152.

Hodson, D. (2006). Pour une approche plus critique au regard du travail pratique en science à l'école. In A. Hasni, Y. Lenoir et J. Lebeaume (dir.). La formation à l'enseignement des sciences et des technologies au secondaire dans le contexte des réformes par compétences (p. 59-96). Sainte-Foy: Presses de l'Université du Québec.

Brock Education, Volume 19, No. 1, Fall 2009, 73-93 
Kang, N.-H. et Wallace, C.S. (2004). Secondary science teachers' use of laboratory activities: linking epistemological beliefs, goals and practices. Science Teacher Education, 89(1), 140-165.

Krueger, R.A. et Casey, M.A. (2000). Focus group. A practical guide for applied research ( $3 \mathrm{e}$ éd.). Thousand Oaks, CA: Sage Publications.

Lang, V. (2005). La profession enseignante en France : permanence et éclatement. In C. Lessard et M. Tardif (dir.), La profession d'enseignant aujourd'hui. Évolutions, perspectives et enjeux internationaux. (p. 157-171). Bruxelles : De Boeck.

Lapointe, J.J. (1992). La conduite d'une étude de besoins en éducation et en formation. Sillery : Presses de l'Université du Québec.

Leach, J., Millar, R., Ryder, J. et Séré, M.-G. (2000). Epistemological understanding in science learning: the consistency of representations across contexts. Learning and Instruction, 10, 497-527.

Lebeaume, J. (2002). L'enseignement régulier de la technologie dans l'hétérogénéité des acteurs et des contextes. Aster, 35, 65-83.

Legault, J.-P. (1993). La gestion disciplinaire de la classe. Montréal : Les Éditions Logiques.

Leloup, S. (2003). L'ennui des lycéens : du manque de motivation au décalage des attentes. Reims: Université de Reims. Document téléaccessible à l'adresse $<$ http://www.pedagopsy.eu/analyse_des_ecarts.htm>. (Consulté le 25 août 2007)

Lessard, C. (2005). Collaboration au travail : norme professionnelle et développement d'une pratique d'enseignement. In D. Biron, M. Cividini et J.-F. Desbiens (dir.), $L a$ profession enseignante au temps des réformes (p. 435-457). Sherbrooke : Éditions du CRP.

Louart, P. (2002). Maslow, Herzberg et les théories du contenu motivationnel. Les cahiers de la recherche du CLAREE, 13. Document téléaccessible à l'adresse $<$ http://claree.univ-lille1.fr/ lecocq/cahiers/Maslow,_Herzberg.pdf> (consulté le 3 septembre 2006)

Malo, A. (2000). Savoirs de formation et savoir d'expérience: un processus de transformation. Éducation et francophonie, XXVIII(2). Document téléaccessible à l'adresse $<$ http//www.acelf.ca>. (Consulté le 3 septembre 2006)

Ménard, L. et Laferrière, T. (2006). La communication pédagogique dans une communauté d'apprenants en réseau: récit de pratique. L'éducation en débat: analyse comparée, 4, 47-62. 
Monfroy, B. (2002). La définition des élèves en difficulté en ZEP : le discours des enseignants de l'école primaire. Revue française de pédagogie, 140(juillet-aoûtseptembre), 34-40.

Morandi, F. (2006). Difficulté d'enseigner, difficulté d'apprendre. In Y. Montoya, J.-P. Martinez et G. Boutin (dir.), L'école actuelle face au changement. Instruire, éduquer ou socialiser (p. 61-72). Québec : Presses de l’Université du Québec.

Nault, T. et Fijalkow, J. (1999). Introduction. La gestion de la classe : d'hier à demain. Revue des sciences de l'éducation, XXV(3), 451-466.

Paldy, L.G. (2003). Forgotten history, ignored research, little progress. Journal of College Science Teaching, 32(7), 422.

Pelletier, G. (2005). De quelques réflexions sur la gestion du changement et sur l'art de diriger le dirigeant. In D. Biron, M. Cividini et J.-F. Desbiens (dir.), La profession enseignante au temps des réformes (p. 89-102). Sherbrooke : Éditions du CRP.

Potvin, P., Fournier, F., Mauffette, Y. et Paradis, P. (2007). Résoudre le paradoxe de la formation de formateurs en contexte de réforme: l'enseignement scientifique et technologique pour le secondaire à l'UQAM-ÉTS. Communication présentée dans le cadre du 75 ${ }^{\mathrm{e}}$ congrès de l'ACFAS, Trois-Rivières, Canada, 7-11 mai.

Pourtois, J.-P. et Desmet, H. (1997). L'éducation postmoderne. Paris : Presses Universitaires de France.

Pourtois, J.-P. et Desmet, H. (2004). L'éducation implicite. Paris : Presses Universitaires de France.

Purseigle, F. (2004). L'engagement professionnel des jeunes agriculteurs, du concept aux représentations. Lexicométrica, 5. Document téléaccessible à l'adresse $<$ http://www.cavi.univ-paris3.fr/lexicometrica/article/numero5/lexicometricapurseigle. pdf> (consulté le 2 mars 2006).

Reiss, M.J. (2004). Students' attitudes towards science: a long-term perspective. Revue canadienne de l'enseignement des sciences, des mathématiques et des technologies, 4(1), 97-109.

Richoux, H. et Beaufils, D. (2005). Conception de travaux pratiques par les enseignants : analyse de quelques exemples de physique en termes de transposition didactique. Didaskalia, 27, 11-39.

Roth, W.-M. (2005). Ethnomethodology and the r/evolution of science education. Revue canadienne de l'enseignement des sciences, des mathématiques et des technologies, 5(2), 185-198. 
Roth, W.-M. (2006). Une approche par la pratique des réformes en enseignement et en apprentissage des sciences. In A. Hasni, Y. Lenoir et J. Lebeaume (dir.), La formation à l'enseignement des sciences et des technologies au secondaire dans le contexte des réformes par compétences (p. 157-191). Québec: Presses de l'Université du Québec.

Shapiro, B. (2004). Studying lifeworlds of science learning: a longitudinal study of changing ideas, contexts, and personal orientations in science learning. Revue canadienne de l'enseignement des sciences, des mathématiques et des technologies, $4(1), 127-147$.

Suits, J.P. (2004). Assessing investigative skill development in inquiry-based and traditional college science laboratory courses. School Science and Mathematics, 104(6), 248-257.

Tardif, J. (2006). L'évaluation des compétences: documenter le parcours de développement. Montréal : Chenelière éducation.

Tardif, M. (2005). Enseigner aujourd'hui : entre l'espace artisanal du travail en classe et le temps des grandes mutations. In D. Biron, M. Cividini et J.-F. Desbiens (dir.), La profession enseignante au temps des réformes (p. 73-89). Sherbrooke : Éditions du CRP.

Thouin, M. (2002). Didactique des sciences, changement conceptuel et formation des maîtres. In R.M.J. Toussaint (dir.), Changement conceptuel et apprentissage des sciences. Recherches et pratiques (p. 157-176). Outremont : Éditions Logiques.

Tsai, C.-C. (2001). The interpretation construction design model for teaching science and its applications to Internet-based instruction in Taiwan. International Journal of Educational Development, 21, 410-415.

Vérin, A. (1998). Enseigner de façon constructiviste, est-ce faisable? Aster, 26, 131-163.

Watkins, M.W et Pacheco, M. (2001). Interobserver agreement in behavioral research: importance and calculation. Journal of Behavioral Education, 10(4), 205-212.

Yvon, F. et Garon, R. (2006). Une forme d'analyse du travail pour développer et connaitre le travail enseignant: l'autoconfrontation croisée. Recherches qualitatives, 26(1), 51-80.

Brock Education, Volume 19, No. 1, Fall 2009, 73-93 